\title{
Salvage interstitial brachytherapy based on computed tomography for recurrent cervical cancer after radical hysterectomy and adjuvant radiation therapy: case presentations and introduction of the technique
}

\author{
Zhong-Shan Liu, MD!, Jie Guo, MD!, Yang-Zhi Zhao, MD², Xia Lin, BS', Bin Chen, MD³, Ming Zhang, MD², \\ Jiang-Ming Li, MD', Xiao-Jun Ren, MD', Bing-Ya Zhang, MD', Tie-Jun Wang, MD, PhD' \\ 'Department of Radiation Oncology, The Second Affiliated Hospital of Jilin University, Changchun, ${ }^{2}$ Cancer Center, The First Affiliated Hospital \\ of Jilin University. Changchun, ${ }^{3}$ Department of Nuclear Medicine, The Third Affiliated Hospital of Jilin University, Changchun, \\ ${ }^{4}$ Department of Radiology, the General Hospital of Hegang Mining Industry Group Co., Ltd, Hegang, ${ }^{5}$ Department of General Surgery. \\ The $313^{\text {th }}$ Hospital of People's Liberation Army. Huludao, China
}

\begin{abstract}
Purpose: Locally recurring cervical cancer after surgery and adjuvant radiotherapy remains a major therapeutic challenge. This paper presents a new therapeutic technique for such patients: interstitial brachytherapy (BT) guided by real-time three-dimensional (3D) computed tomography (CT).

Material and methods: Sixteen patients with recurrent cervical cancer after radical surgery and adjuvant external-beam radiotherapy (EBRT) were included in this study. These patients underwent high-dose-rate (HDR) interstitial BT with free-hand placement of metal needles guided by real-time 3D-CT. Six Gy in 6 fractions were prescribed for the high-risk clinical target volume (HR-CTV). $\mathrm{D}_{90}$ and $\mathrm{D}_{100}$ for HR-CTV of BT, and the cumulative $\mathrm{D}_{2 \mathrm{cc}}$ for the bladder, rectum, and sigmoid, including previous EBRT and present BT were analyzed. Treatment-related complications and 3-month tumor-response rates were investigated.

Results: The mean $\mathrm{D}_{90}$ value for HR-CTV was $52.5 \pm 3.3 \mathrm{~Gy}$. The cumulative $\mathrm{D}_{2 \mathrm{cc}}$ for the bladder, rectum, and sigmoid were $85.6 \pm 5.8,71.6 \pm 6.4$, and $69.6 \pm 5.9$ Gy, respectively. The mean number of needles was $6.1 \pm 1.5$, with an average depth of $3.5 \pm 0.9 \mathrm{~cm}$ for each application. Interstitial BT was associated with minor complications and passable tumor-response rate.

Conclusions: Interstitial BT guided by real-time 3D-CT for recurrent cervical cancer results in good dose-volume histogram (DVH) parameters. The current technique may be clinically feasible. However, long-term clinical outcomes should be further investigated.
\end{abstract}

Key words: cervical cancer, computed tomography, interstitial brachytherapy, recurrence.

\section{Purpose}

Patients with recurrent cervical cancers represent a therapeutic challenge. Choosing an optimal treatment approach for recurrent patients who have undergone radical hysterectomy, adjuvant radiation therapy, and possibly, concurrent chemotherapy is a difficult task. Surgical resection with pelvic exenteration is considered the treatment of choice, but this is associated with a severe treatment-related morbidity $[1,2,3]$. Moreover, some patients with recur- rence of cancer on their pelvic walls are considered inoperable. Re-irradiation by using external beam radiotherapy (EBRT) alone may be difficult for treatment of pelvic recurrences, due to the high risk of complications $[4,5]$.

Another treatment option for patients with recurrent tumors is brachytherapy (BT). Traditional intracavitary $\mathrm{BT}$, using vaginal cylinder and ovoid pairs, may be sufficient for small and superficial tumors [6,7]. However, recurrent cervical cancers with bulky tumors, anatomical distortion, or pelvic wall involvement are difficult to
Address for correspondence: Tie-Jun Wang, MD, Department of Radiation Oncology, The Second Affiliated Hospital of Jilin University, 218 Zi Qiang Street, Changchun 130041, China, phone: +86 0431-88796178, fax: +86 0431-88796178, « e-mail: wangtiejunjlu@163.com
Received: 19.07 .2016

Accepted: 07.10 .2016

Published: 31.10 .2016 
cure by this technique, due to inadequate target coverage during intracavitary BT $[8,9]$. Interstitial BT, which has the ability to tailor the dose to the target, and allow for wider tumor coverage, is more advantageous than conventional intracavitary BT $[10,11,12,13]$.

In this study, we introduce real-time interstitial BT guided by three-dimensional (3D) computed tomography $(\mathrm{CT})$ as a treatment approach for recurrent cervical cancer after radical hysterectomy and adjuvant radiation therapy. This new technique may provide a simple and effective clinical treatment modality.

\section{Material and methods}

\section{Patients and treatment}

We analyzed consecutive patients who experienced isolated pelvic recurrences of cervical carcinoma, and had previously received initial radical surgery and adjuvant EBRT in our department, between December 2014 and May 2016. These patients underwent a systematic examination, including a pelvic MRI (magnetic resonance imaging) (1.5 T; $5 \mathrm{~mm}$ sections), a whole-body CT or positron emission tomography - computed tomography (PET-CT), and a gynecological examination. In all cases, either the recurrent tumors were inoperable or the patients refused surgical resection due to the possibility of serious complications. We performed re-irradiation on all these patients by using interstitial BT. A total of six high-dose-rate (HDR) interstitial BT procedures, using ${ }^{192}$ Ir and implantation with metal needles, were carried out for 4-5 days per fraction. This study and the treatment procedure used for patients was approved by the ethics committee of our hospital. All patients signed informed written consents before undergoing BT.

\section{Needles implantation under real-time 3D-CT guidance}

Each needle implantation procedure was performed with the patient under subarachnoid anesthesia in the lithotomy position. Interstitial metal needles (length $=$
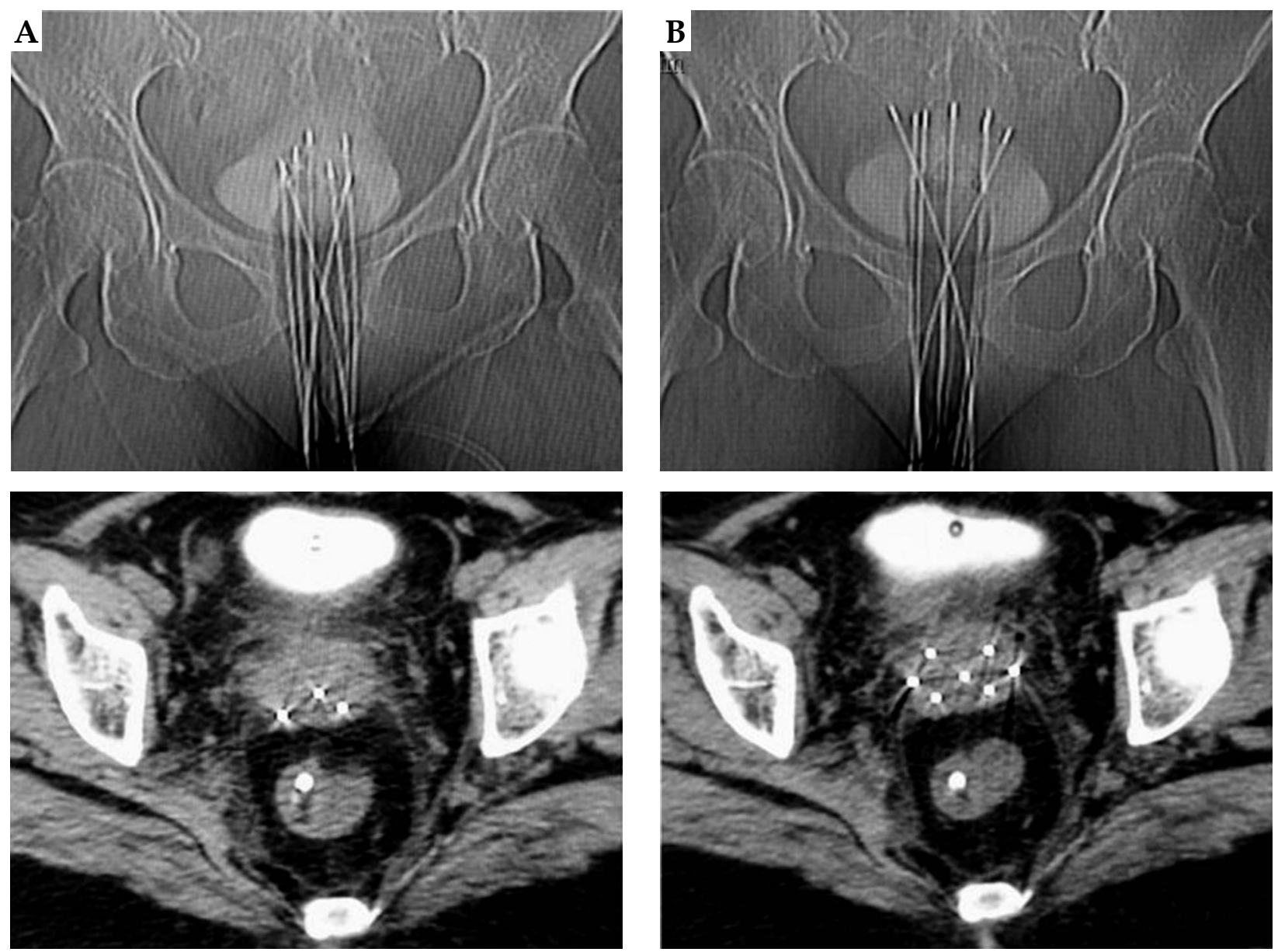

Fig. 1. Representative example of interstitial needle implantation guided by real-time three-dimensional (3D) computed tomography $(\mathrm{CT})$, for recurrent cervical cancer. Five interstitial metal needles were inserted by free-hand placement into the central tumor parallel to the vagina, and two interstitial metal needles were inserted into the distal extension at certain angles to the vagina at a depth of approximately $10 \mathrm{~mm}$, as a preliminary implantation. The direction and depth of the seven needles was adjusted until satisfactory positioning was observed by multiple CT scans. A) Preliminary distribution of the needle position on the poster anterior radiograph (upper) and the axial CT image (lower) before adjustment (B). Final distribution of the needle position on the poster anterior radiograph (upper) and axial CT image (lower) after adjustment 
$16 \mathrm{~cm}$, diameter $=1.3 \mathrm{~mm}$, Elekta company, Elekta $\mathrm{AB}$, Stockholm, Sweden) were inserted into the recurrent tumor at a depth of approximately $10 \mathrm{~mm}$ as a preliminary implantation, according to T2-weighted MRI and clinical gynecological examination before administration of BT. Specifically, metal needles were placed parallel to the vagina into the central tumor, and some tilted needles at different angles to the vagina were placed into the parametrial or lateral pelvic sidewall extensions, transvaginally. In case of a central tumor with anterior or posterior vaginal wall involvement, the needles were placed into the central tumor parallel to the vagina, but the inserted needles were close to the vaginal wall for optimal coverage of vaginal wall involvement. Vaginal packing was done by using gauze to push aside the rectum and the bladder, and to stabilize the metal needles.

After the initial implantation of needles, the bladder was filled with $50 \mathrm{ml}$ of diluted urografin (dilution, $1: 20$ ) through a urinary catheter. Next, the process of final needles-implantation guided by real-time 3D-CT was performed. The direction and the depth of the metal needles were adjusted after every CT scan (Philips Healthcare, Eindhoven, The Netherlands, $4 \mathrm{~mm}$ slice intervals). Adjustment of needles was performed repeatedly through multiple CT scans until satisfactory distribution was achieved, including accurate needle-insertion in the recurrent tumor, and symmetrical needle-distribution in the tumor at a $1 \mathrm{~cm}$ distance from the central axis (Figure 1). Eligible dose-volume histogram (DVH) parameters for the target volume and the organs at risk (OAR) could be acquired with satisfactory distribution of needles in subsequent treatment planning.

\section{Contouring and treatment planning}

Using CT images, the high-risk clinical target volume (HR-CTV) that encompassed the volume occupied by the recurrent tumors, and OAR, including the bladder, rectum, and sigmoid, were contoured. Additionally, the T2-weighted MRI images and clinical gynecological examinations were also used as reference for tumor delineation.

The dose for the target volume during BT and the cumulative dose of OAR, obtained by combining previous EBRT and present BT, were all converted to the equivalent dose in $2 \mathrm{~Gy}\left(\mathrm{EQD}_{2}\right)$ by applying the linear quadratic model using an $\alpha / \beta=10$ for target coverage, and $\alpha / \beta$ $=3$ for normal tissue. The final dose objective was HRCTV $\mathrm{D}_{90}$ (the minimum dose delivered to $90 \%$ of the target volume) $\geq 50$ Gy $\mathrm{EQD}_{2}$ for $\mathrm{BT}$; the cumulative $\mathrm{D}_{2 \mathrm{cc}}$ (the minimal dose for the most irradiated $2 \mathrm{~cm}^{3}$ ) for the bladder $\leq 90$ Gy $\mathrm{EQD}_{2}$; the cumulative $\mathrm{D}_{2 \mathrm{cc}}$ for the rectum and sigmoid $\leq 75 \mathrm{~Gy} \mathrm{EQD}_{2}$. For BT planning, needle applicators were initially reconstructed and registered to CT images (Oncentra Brachytherapy Treatment Planning System, Elekta). Subsequently, an inverse-planning simulated annealing (IPSA) was used with a 6 Gy prescription dose for the HR-CTV. Dwell positions and dwell weights of the radioactive source were manually adjusted until the dose distribution was optimally matched to our dose requirement when the first treatment plan based on IPSA did not meet the dose requirement (Figure 2).

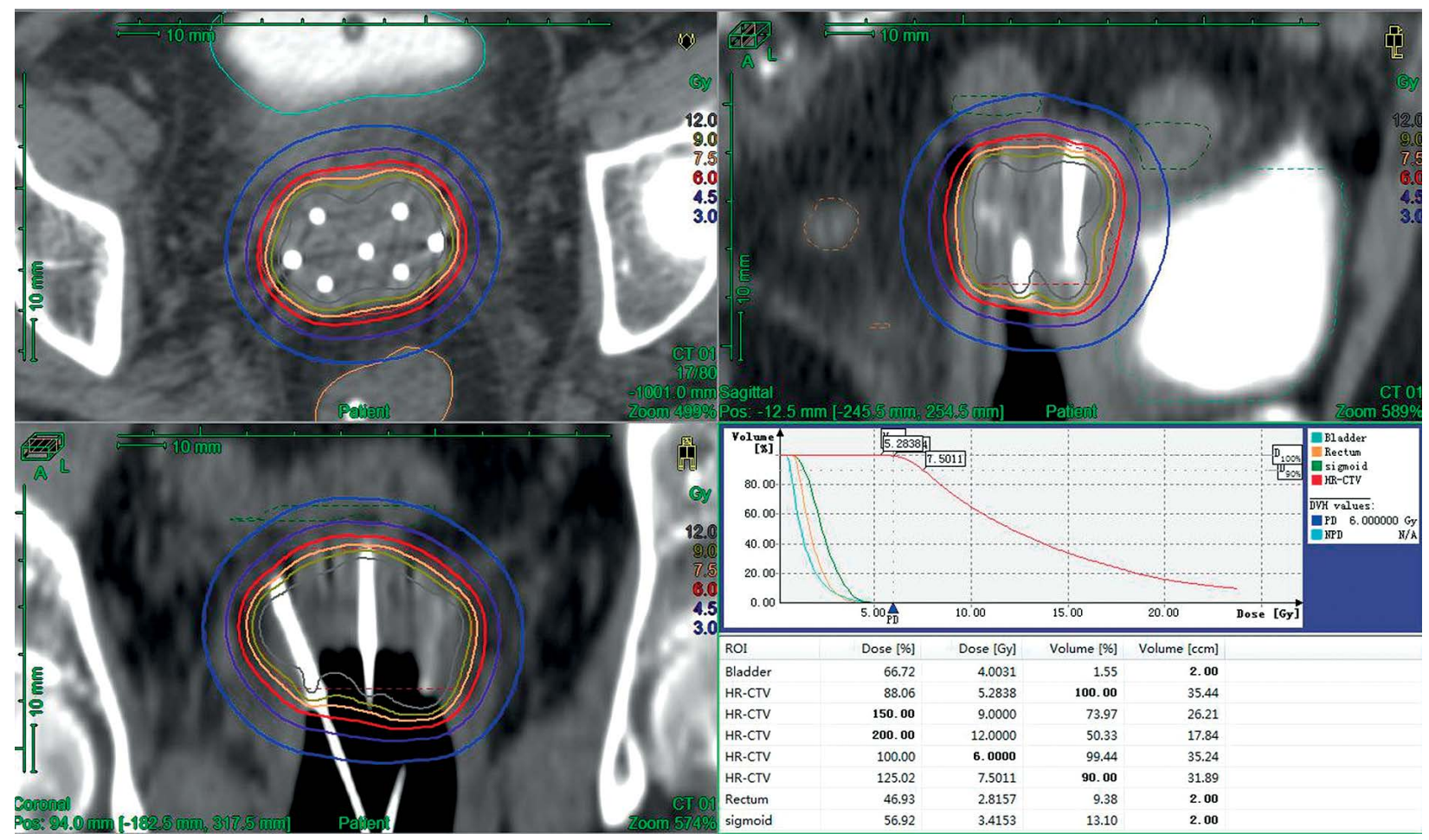

Fig. 2. Representative treatment planning of interstitial brachytherapy, guided by computed tomography, for recurrent cervical cancer. A dose of 6 Gy was prescribed for high-risk clinical target volume (HR-CTV) by using seven interstitial metal needles inserted to the recurrent tumor 


\section{Results}

\section{Initial and recurrence tumor characteristics}

Sixteen patients with recurrent cervical cancer were enrolled into this study. All patients had undergone radical hysterectomy with pelvic lymph node dissection, and post-hysterectomy adjuvant EBRT with a total dose of 45 Gy administered as 1.8 Gy in 25 fractions, using either the four-field or intensity-modulated radiation-therapybased 3D-CT. Twelve patients received 5 cycles of weekly cisplatin chemotherapy (dose, $40 \mathrm{mg} / \mathrm{m}^{2}$ ) and none of the patients were treated with initial vaginal BT. A summary of tumor characteristics at initial treatment and at the time of recurrence is presented in Table 1.

\section{Dose-volume histogram parameters}

Individual recurrent tumor characteristics and DVH parameters are shown in Table 2. Dose-volume histogram parameters related to HR-CTV $\mathrm{D}_{90}$ showed that our inter-

Table 1. Initial and recurrent tumor characteristics

\begin{tabular}{|c|c|}
\hline Characteristic & Value \\
\hline Median (y) (range) & $52.4(33-76)$ \\
\hline \multicolumn{2}{|l|}{ Initial FIGO stage (\%) } \\
\hline $\mathrm{IB1}$ & $2(13)$ \\
\hline IB2 & $5(31)$ \\
\hline$\| \mathrm{A} 1$ & $5(31)$ \\
\hline IIA2 & $4(25)$ \\
\hline \multicolumn{2}{|l|}{ Histology (\%) } \\
\hline Squamous cell carcinoma & $14(88)$ \\
\hline Adenocarcinoma & $1(6)$ \\
\hline Adenosquamous carcinoma & $1(6)$ \\
\hline \multicolumn{2}{|l|}{ Pathological nodes } \\
\hline No & 6 \\
\hline Yes & 10 \\
\hline \multicolumn{2}{|l|}{ Recurrence interval (months) } \\
\hline$<12$ & 9 \\
\hline$>12$ & 7 \\
\hline \multicolumn{2}{|l|}{ Sites of recurrence } \\
\hline Central only & 8 \\
\hline Central and pelvic wall & 8 \\
\hline \multicolumn{2}{|l|}{ OAR involvement } \\
\hline Bladder & 2 \\
\hline Rectum & 1 \\
\hline
\end{tabular}

FIGO - International Federation of Gynecology and Obstetrics, OAR - organs at risk stitial technique resulted in a $\mathrm{D}_{90}$ of $\geq 50$ Gy in $93.4 \%$ patients. The target volume dose was unsatisfactory only for one patient who had central and pelvic wall extensions. High-risk clinical target volume $\mathrm{D}_{90}$ for this patient was 45.1 Gy, which could be mainly attributed to dose constraint for the sigmoid $\left(\mathrm{D}_{2 \mathrm{cc}}\right.$ for sigmoid was 78.9 Gy for this patient). The results of DVH analysis for OAR showed that two patients had $\mathrm{D}_{2 \mathrm{cc}}$ of $>90 \mathrm{~Gy}$ for the bladder, and one patient had a $\mathrm{D}_{2 \mathrm{cc}}$ of $>75 \mathrm{~Gy}$ for the rectum, due to involvement of bladder and rectum, respectively.

Table 3 shows the mean DVH parameters derived for all patients and for the patients with different sites of recurrence, respectively. A double-sided $t$ test for two independent samples was used to compare the mean values of DVH parameters between patients with central recurrence and patients with central and pelvic wall involvement. A significant decrease in the mean $D_{90}$ value for HR-CTV was seen from the cases of central recurrence to those with central and pelvic wall involvement $(p=0.04)$. However, no statistically significant differences in the mean $D_{100}$ value for HR-CTV, as well as the average $D_{2 c c}$ value for the OAR were noted between the two.

\section{Characteristics of free metal needles}

In all, 588 free needles were used for 96 independent interstitial BT procedures, and $95.9 \%$ of all implanted needles were useful for treatment. The mean number of free needles was $6.1 \pm 1.5$ for each application, the mean implantation depth of the needles was $3.5 \pm 0.9 \mathrm{~cm}$, and the mean implanted length of the needles beyond the tumor was $0.4 \pm 0.3 \mathrm{~cm}$.

Mean time from preliminary insertion of needles to completion of CT scans for each interstitial implantation was $30.9 \pm 8.6$ minutes, and the mean number of CT scans was $2.3 \pm 1.2$ for every placement of metal needles.

\section{Complications and tumor response}

During real-time 3D-CT guided interstitial needle implantation, one patient with bladder involvement had a bladder perforation, and two patients had slight perforation of the intestine. These patients did not require any additional treatment because no clinical effects were observed. However, the radioactive source could not be placed in the perforated area during subsequent treatment planning. Minimal post-procedure edema occurred in one patient. No case of obvious infection or severe bleeding with requirement for transfusion was founded during or immediately after implantation. At the 3 months' follow-up, one patient had bloody stools, which was resolved by treatment with clyster with styptic, hormones, and antibiotics. No serious complication was observed in the vaginal mucosa.

Initial response, defined as complete remission (CR) and partial remission (PR), was found in 13 (81.3\%) patients, according to pelvic MRI findings and clinical gynecological examination at 3 months after interstitial BT. Of all the patients with initial responses, $6(37.5 \%)$ exhibited CR, and 7 (43.8\%) PR. The remaining three (18.7\%) patients showed stable disease (SD) at 3 months after interstitial BT. No patient experienced progressive disease (PD). 
Table 2. Recurrent tumor characteristics and dose-volume histogram parameters for all patients

\begin{tabular}{|c|c|c|c|c|c|c|c|c|}
\hline Patient & $\begin{array}{l}\text { Sites of } \\
\text { recurrence }\end{array}$ & $\begin{array}{c}\text { HR-CTV } \\
\text { volume }\left[\mathrm{cm}^{3}\right]\end{array}$ & $\begin{array}{c}\text { OAR } \\
\text { involvement }\end{array}$ & $\begin{array}{c}\text { HR-CTV D } \\
{[G y]}\end{array}$ & HR-CTV $D_{100}$ & Bladder $D_{2 c c}$ & Rectum $D_{2 c c}$ & Sigmoid $D_{2 c c}$ \\
\hline 1 & Central only & 37.1 & None & 59.4 & 33.2 & 80.2 & 67.8 & 73.1 \\
\hline 2 & Central only & 99.3 & None & 55.1 & 30.1 & 89.3 & 71.6 & 72.0 \\
\hline 3 & Central only & 85.8 & None & 52.8 & 31.8 & 76.9 & 72.3 & 55.8 \\
\hline 4 & Central only & 47.9 & None & 54.6 & 26.8 & 86.0 & 60.6 & 73.3 \\
\hline 5 & Central only & 34.6 & None & 57.2 & 28.8 & 83.9 & 74.5 & 61.0 \\
\hline 6 & Central only & 43.9 & None & 50.4 & 25.1 & 88.0 & 70.3 & 70.1 \\
\hline 7 & Central only & 50.8 & None & 51.7 & 26.3 & 89.1 & 71.0 & 74.7 \\
\hline 8 & Central only & 118.7 & Bladder & 51.9 & 30.2 & 94.3 & 73.9 & 68.0 \\
\hline 9 & $\begin{array}{l}\text { Central and } \\
\text { pelvic wall }\end{array}$ & 60.8 & None & 50.8 & 25.9 & 83.0 & 64.8 & 74.0 \\
\hline 10 & $\begin{array}{l}\text { Central and } \\
\text { pelvic wall }\end{array}$ & 43.0 & None & 45.1 & 22.4 & 74.0 & 68.3 & 78.9 \\
\hline 11 & $\begin{array}{l}\text { Central and } \\
\text { pelvic wall }\end{array}$ & 66.1 & None & 55.4 & 29.7 & 87.3 & 73.8 & 72.6 \\
\hline 12 & $\begin{array}{l}\text { Central and } \\
\text { pelvic wall }\end{array}$ & 35.6 & None & 50.6 & 24.7 & 85.7 & 68.0 & 74.1 \\
\hline 13 & $\begin{array}{l}\text { Central and } \\
\text { pelvic wall }\end{array}$ & 68.4 & None & 51.6 & 25.2 & 82.1 & 74.6 & 65.1 \\
\hline 14 & $\begin{array}{l}\text { Central and } \\
\text { pelvic wall }\end{array}$ & 56.5 & None & 50.3 & 29.7 & 88.9 & 69.1 & 64.7 \\
\hline 15 & $\begin{array}{l}\text { Central and } \\
\text { pelvic wall }\end{array}$ & 125.1 & Bladder & 52.3 & 29.6 & 96.6 & 73.8 & 65.6 \\
\hline 16 & $\begin{array}{l}\text { Central and } \\
\text { pelvic wall }\end{array}$ & 98.2 & Rectum & 50.2 & 24.3 & 85.0 & 90.9 & 70.4 \\
\hline
\end{tabular}

OAR - organs at risk, CTV - clinical target volume, HR - high risk, IR - intermediate risk, HR-CTV-volume was generated based on $C T$, $D_{90}$ and $D_{100}-t$ the minimum dose delivered to 90 and $100 \%$ of the target volume, respectively; $D_{2 c c}$ - the minimal dose for the most irradiated $2 \mathrm{~cm}^{3}$ HR-CTV volume was generated based on CT

The dose for the OAR $D_{2 c c}$ are the cumulative total from previous external beam radiotherapy and present brachytherapy Dose values are converted to the equivalent dose in $2 \mathrm{~Gy}\left(E Q D_{2} ; \alpha / \beta=10\right.$ Gy for tumor, $\alpha / \beta=3$ Gy for normal tissue)

Table 3. Dose-volume histogram parameters for entire patients and the patients with different sites of recurrence, respectively

\begin{tabular}{lccc} 
Parameter & Entire population $(n=16)$ & Central recurrence $(n=8)$ & Central and pelvic wall involvement $(n=8)$ \\
\hline HR-CTV $D_{90}$ & $52.5 \pm 3.3 \mathrm{~Gy}$ & $54.1 \pm 3.0 \mathrm{~Gy}$ & $50.8 \pm 2.9 \mathrm{~Gy}(p=0.04)^{\star}$ \\
\hline HR-CTV $D_{100}$ & $27.7 \pm 3.1 \mathrm{~Gy}$ & $29.0 \pm 2.8 \mathrm{~Gy}$ & $26.4 \pm 2.9 \mathrm{~Gy}$ \\
\hline Bladder $\mathrm{D}_{2 \mathrm{Cc}}$ & $85.6 \pm 5.8 \mathrm{~Gy}$ & $86.0 \pm 5.5 \mathrm{~Gy}$ & $85.3 \pm 6.4 \mathrm{~Gy}$ \\
\hline Rectum $\mathrm{D}_{2 \mathrm{cc}}$ & $71.6 \pm 6.4 \mathrm{~Gy}$ & $70.3 \pm 4.4 \mathrm{~Gy}$ & $72.9 \pm 8.1 \mathrm{~Gy}$ \\
\hline Sigmoid $\mathrm{D}_{2 c \mathrm{cc}}$ & $69.6 \pm 5.9 \mathrm{~Gy}$ & $68.5 \pm 6.7 \mathrm{~Gy}$ & $70.7 \pm 5.2 \mathrm{~Gy}$
\end{tabular}

$C T V$ - clinical target volume, HR - high risk, IR - intermediate risk, $D_{90}$ and $D_{100}$ - the minimum dose delivered to 90 and $100 \%$ of the target volume, respectively, $D_{2 c c}$ - the minimal dose for the most irradiated $2 \mathrm{~cm}^{3}$

Dose values are expressed as mean \pm standard deviation (SD) equivalent doses for 2 Gy fractions $\left(E Q D_{2} ; \alpha / \beta=10\right.$ Gy for tumor, $\alpha / \beta=3$ Gy for normal tissue) *Statistically significant values compared with HR-CTV $D_{90}$ for the patients with central recurrence (statistical significance was considered when $p<0.05$ )

\section{Discussion}

We studied the dosimetric results for treatment of 16 patients with recurrent cervical cancer who had received previous radical surgery and adjuvant EBRT with a novel form of interstitial BT that used free-hand placement of metal needles under guidance of real-time 3D-CT. Cervical cancer with parametrial extensions after surgery often reoccurs [14]. Salvage interstitial BT is an effective treatment strategy [15], especially for inoperable pelvic recurrence. However, the prescribed dose needs to 
be strictly limited to avoid serious complications in OAR. A total dose of 40-50 Gy for BT alone might be effective and well tolerated [16]. Nag et al. reported the clinical outcomes of using interstitial BT for re-irradiation, with a median dose of 50 Gy for patients with recurrent gynecologic malignancies [17]. This study showed a 5-year local control of $54 \%$. In the current study, a HR-CTV $\mathrm{D}_{90}$ of $\geq 50$ Gy produced excellent results in $>93 \%$ of patients. High-risk clinical target volume $\mathrm{D}_{90}$ of the only one patient with central and pelvic wall extension was less than $50 \mathrm{~Gy}$. This can be attributed mainly to dose constraint of the sigmoid. A compromise on the actual delivered HR-CTV dose in this patient had to be done in order to avoid potential radiation-related intestinal perforation. Dose constraints for OAR, with $\mathrm{D}_{2 \mathrm{cc}} \leq 90 \mathrm{~Gy}$ for the bladder, and $D_{2 c c} \leq 75$ Gy for the rectum and sigmoid, were recommended by the American Brachytherapy Society (ABS) [18]. In this study, $D_{2 c c}$ for OAR in all patients reached $43.2 \mathrm{~Gy} \mathrm{EQD}_{2}$ because of prior pelvic irradiation. Resultantly, for the present protocol of interstitial BT, $\mathrm{D}_{2 \mathrm{cc}}$ for the bladder of $\leq 46.8 \mathrm{~Gy} \mathrm{EQD}_{2}$, and $\mathrm{D}_{2 \mathrm{cc}}$ for the rectum and sigmoid of $\leq 31.8 \mathrm{~Gy} \mathrm{EQD}_{2}$ were decided. It was important to consider the cumulative $\mathrm{EQD}_{2}$ of $\mathrm{OAR} \mathrm{D}_{2 \mathrm{cc}}$ combining previous EBRT and present BT, because this was a setting for re-irradiation. Our results indicate that the cumulative $\mathrm{EQD}_{2}$ of $\mathrm{OAR} \mathrm{D}_{2 \mathrm{cc}}$ were generally eligible, except in two patients with bladder involvement, one patient with rectum involvement, and one patient with adherence of the sigmoid to the tumor. Although the mean HR-CTV $D_{90}$ of patients with central and pelvic wall involvement was poorer than that of the patients with central recurrence, it can be mainly attributed to the difficulty in distributing needles effectively in the distal tumor; the mean HR-CTV $D_{90}$ for these patients with central and pelvic wall involvement still reached $50 \mathrm{~Gy}$. Our current clinical experience suggests that this technique has the potential to ensure relatively satisfactory dose-coverage to the HR-CTV, and reduce the irradiated dose of OAR in patients with recurrent cervical cancer.

Traditionally, interstitial BT for recurrent cervical cancer was performed using the classic transperineal interstitial applicators, including Syed-Neblett butterfly template $[19,20]$ and Martinez Universal Perineal Interstitial Template (MUPIT) (Varian Medical Systems, Inc., Palo Alto, CA, USA) [9,21]. Limitations of both these techniques include the following: inaccuracy of needle positioning resulting from a long distance between the template and the tumor, leaving the applicators in place for a few days after implantation, and high risk of complications due to the need for multiple needles.

Free-hand placement of needles is conventionally used for recurrent tumors in many centers. However, accurate free-hand placement is difficult without guidance by realtime images. Guidance by real-time 3D-CT may overcome the shortcomings of traditional free needles. In the present study, real-time 3D-CT guided placement of the needles is performed, to ensure proper needle-positioning for effective target dose coverage. It is the most important procedure for adjusting the direction and depth of metal needles repeatedly, until satisfactory results are obtained through multiple CT scans. In our clinical experience, the metal needles parallel to the vagina as well as at different angles to the vagina were used, according to various case scenarios of recurrence. Typically, the needles did not need to be inserted into the vaginal wall involvement but close to it; greater angulation of vagina and depth of needles were required in case of severe lateral involvement. Two experienced gynecologic radiation oncologists performed the entire process of needle implantation. Real-time 3D-CT guidance of the free needles provides some advantages, including flexibility and accuracy in needle positioning.

In the present study, more than $95 \%$ of all inserted needles were used for BT treatment. This indicates great efficiency of needles in performing dose-coverage of the target volume. The mean number of needles needed for each BT was significantly fewer than that required in a previous study, in which a median of 14 (range, 8-22) interstitial needles had been implanted using MUPIT applicator [22]. Undoubtedly, risks for complications, such as bleeding, increase proportionally with the number of needles. Therefore, this real-time CT guided method of needle implantation may present lower risks for patients than the classic transperineal interstitial method. Mean time of metal needle implantation in this study were relatively short, indicating the high efficiency and procedural simplicity of this technique. In addition, the metal needle is reusable and is available in most centers; this suggests that our technique is low-cost, easy to spread, and especially helpful for departments with limited resources.

The risk of complications such as perforation, bleeding, and infection for interstitial BT, during or immediately after implantation, may be inevitable $[23,24,25]$. In this study, a clinically insignificant bladder perforation was found in one patient with bladder involvement. Moreover, intestine perforation without any clinical effect occurred in other two patients. Our technique shows a low rate of perforation during implantation, which could be attributed to the use of real-time CT guidance. In our clinical practice, needle implantation is always performed cautiously with an adjustment of less than $1 \mathrm{~cm}$ depth after every CT scan to reduce occurrence of perforationrelated complications. Incidence rate of post-procedure edema was low, probably because the number of free needles was low for each BT, and needles were inserted transvaginally, not transperineally. Slight bleeding often occurred due to the insertion of metal needles. However, as the needles were very thin, gauze padding was sufficient to resolve the bleeding.

At the 3 months' follow-up, we diagnosed treatmentrelated rectum morbidity, but during later follow-ups, the adverse effect had been resolved. The 3 month tumor-response rate was excellent; initial response was more than $80 \%$. These results indicate that our technique was well tolerated by patients and exhibited an excellent initial curative effect.

\section{Conclusions}

Salvage interstitial BT with placement of free needles, under real-time 3D-CT guidance, is a particularly appealing treatment option for inoperable pelvic recurrence of 
cervical carcinoma after radical hysterectomy and pelvic irradiation. However, the long-term curative effect and late toxicities of the current technique need to be evaluated further in future studies.

\section{Disclosure}

Authors report no conflict of interest.

\section{References}

1. Maggioni A, Roviglione G, Landoni F et al. Pelvic exenteration: ten-year experience at the European Institute of Oncology in Milan. Gynecol Oncol 2009; 114: 64-68.

2. Berek JS, Howe C, Lagasse LD et al. Pelvic exenteration for recurrent gynecologic malignancy: Survival and morbidity analysis of the 45-year experience at ucla. Gynecol Oncol 2005; 99: 153-159.

3. Miller B, Morris M, Rutledge F et al. Aborted exenterative procedures in recurrent cervical cancer. Gynecol Oncol 1993; 50: 94-99.

4. Ng MK, Leong $\mathrm{T}$, Heriot AG et al. Once-daily reirradiation for rectal cancer in patients who have received previous pelvic radiotherapy. J Med Imaging Radiat Oncol 2013; 57: 512-518.

5. Russell AH, Koh WJ, Markette K et al. Radical reirradiation for recurrent or second primary carcinoma of the female reproductive tract. Gynecol Oncol 1987; 27: 226-232.

6. Hille A, Weiss E, Hess CF. Therapeutic outcome and prognostic factors in the radiotherapy of recurrences of cervical carcinoma following surgery. Strahlenther Onkol 2003; 179: 742-747.

7. Okuma K, Yamashita H, Kobayashi R et al. A study of highdose-rate intracavitary brachytherapy boost for curative treatment of uterine cervical cancer. J Contemp Brachytherapy 2015; 7: 128-134.

8. Jensen PT, Roed H, Engelholm SA et al. Pulsed dose rate (PDR) brachytherapy as salvage treatment of locally advanced or recurrent gynecologic cancer. Int J Radiat Oncol Biol Phys 1998; 42: 1041-1047.

9. Gupta AK, Vicini FA, Frazier AJ et al. Iridium-192 transperineal interstitial brachytherapy for locally advanced or recurrent gynecological malignancies. Int J Radiat Oncol Biol Phys 1999; 43: 1055-1060.

10. Badakh DK, Grover AH. Reirradiation with high-dose-rate remote afterloading brachytherapy implant in patients with locally recurrent or residual cervical carcinoma. J Cancer Res Ther 2009; 5: 24-30.

11. Kotsuma T, Yoshida K, Yamazaki $\mathrm{H}$ et al. Preliminary results of magnetic resonance imaging-aided high-dose-rate interstitial brachytherapy for recurrent uterine carcinoma after curative surgery. J Radiat Res 2011; 52: 329-334.

12. Prempree T. Parametrial implant in stage III B cancer of the cervix. III. A five-year study. Cancer 1983; 52: 748-750.

13. Nandwani PK, Vyas RK, Neema JP et al. Retrospective analysis of role of interstitial brachytherapy using template (MUPIT) in locally advanced gynecological malignancies. J Cancer Res Ther 2007; 3: 111-115.

14. Teke F, Yöney A, Teke M. Evaluation of outcome and prognostic factors in 739 patients with uterine cervix carcinoma: a single institution experience. Contemp Oncol (Pozn) 2015; 19: 130-136.

15. Yoshida K, Yamazaki H, Kotsuma T et al. Treatment results of image-guided high-dose-rate interstitial brachytherapy for pelvic recurrence of uterine cancer. Brachytherapy 2015; 14: $440-448$.

16. Greven KM. Interstitial radiation for recurrent cervix or endometrial cancer in the suburethral region. Int J Radiat Oncol Biol Phys 1998; 41: 831-834.
17. Nag S, Selman AE, Martinez-Monge R. Interstitial reirradiation as salvage treatment for previously irradiated patients with recurrent gynecologic malignancies. J Brachytherapy Int 1998; 14: 49-57.

18. Viswanathan AN, Beriwal S, De Los Santos JF et al. American Brachytherapy Society consensus guidelines for locally advanced carcinoma of the cervix. Part II: High-dose-rate brachytherapy. Brachytherapy 2012; 11: 47-52.

19. Amsbaugh MJ, Bhatt N, Hunter T et al. Computed tomography planned interstitial brachytherapy for recurrent gynecologic cancer. Brachytherapy 2015; 14: 600-605.

20. Stock RG, Chan $K$, Terk $M$ et al. A new technique for performing Syed-Neblett template interstitial implants for gynecologic malignancies using transrectal-ultrasound guidance. Int J Radiat Oncol Biol Phys 1997; 37: 819-825.

21. Martinez A, Edmundson GK, Cox RS et al. Combination of external beam irradiation and multiple-site perineal applicator (MUPIT) for treatment of locally advanced or recurrent prostatic, anorectal, and gynecologic malignancies. Int J Radiat Oncol Biol Phys 1985; 11: 391-398.

22. Fokdal L, Tanderup K, Nielsen SK et al. Image and laparoscopic guided interstitial brachytherapy for locally advanced primary or recurrent gynaecological cancer using the adaptive GEC ESRO target concept. Radiother Oncol 2011; 100: 473-479.

23. Fokdal L, Tanderup K, Hokland SB et al. Clinical feasibility of combined intracavitary/interstitial brachytherapy in locally advanced cervical cancer employing MRI with a tandem/ring applicator in situ and virtual preplanning of the interstitial component. Radiother Oncol 2013; 107: 63-68.

24. Hughes-Davies L, Silver B, Kapp DS. Parametrial interstitial brachytherapy for advanced or recurrent pelvic malignancy: The Harvard/Stanford experience. Gynecol Oncol 1995; 58: 24-27.

25. Martinez A, Cox RS, Edmundson GK. A multiple-site perineal applicator (MUPIT) for treatment of prostatic, anorectal, and gynecologic malignancies. Int J Radiat Oncol Biol Phys 1984; 10: 297-305. 